\title{
Environmental Flow Assessment of Hewa Khola A and Lower Hewa Khola Hydropower Projects in Nepal
}

\author{
Narayanhari Rijal, Hari Krishna Shrestha and Bert Bruins
}

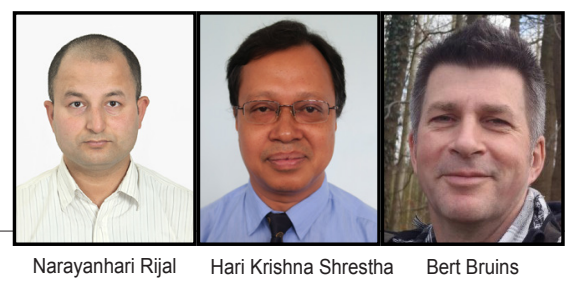

Abstract: One of the elements of sustainable hydropower development is the release of the environmental flows (Eflows). This Eflows is meant to ensure that prior use(r)s of the river water are respected and that essential riverine ecosystem functions are maintained. This paper presents the results of an e-flows assessment, using the hydrological index method, of Hewa Khola A and Lower Hewa Khola Hydropower Projects, in Phidim, Nepal. These projects are being developed in the tributaries of Tamor River in Eastern part of Nepal. The e-flows assessment results are compared with the current releases made from the projects in operation. Current operations are prone to lead to severe degradation and social conflict. Based on an evaluation of the effects of a higher Eflows release on the power production, the paper concludes with recommendations for appropriate Eflow releases, and thus for socially and environmentally sustainable operations of the hydropower projects.

Keywords: Sustainable hydropower development, environmental flows assessment, riverine ecosystem, downstream water uses, Nepal

\section{Introduction}

Tepal has a huge potential for hydropower development, because the country is endowed with a large number of snow fed perennial rivers with topographical variation within a short stretch. After the introduction of Hydropower Development Policy, 2001, there has been active involvement of the private sector in hydropower development. However, it is only recently that sustainable operations of the hydropower projects became a priority.

Environmental assessment and environmental flows are an important aspect for sustainable hydropower development. Environmental Flow refers to the right quantity, quality and timing of water that needs to be released in the dewatered section of river so as to mimic the natural river flow and maintain and sustain existing riverine ecosystem services. Environmental Flow Assessment is a useful tool for defining ecological flow requirements in a riverine ecosystem. Various methods have been developed to determine this ecological flow requirement depending on the type of project, location of project and prevailing environmental regulations in the respective country or region. Widely used methodologies for Eflow Assessment are: Hydrological Index Method, Hydraulic Rating Methods, Habitat Simulation Methods and the Holistic Methods.

This paper outlines the current scenario of environmental release through study of recent hydropower projects, ensures the compliance of the projects with Nepal National regulations and performs an e-flows assessment using the hydrological index method. Eflow Assessment is thus carried out for recently completed Hewa Khola A HEP and an underconstruction Lower Hewa Khola HPP.

\section{Materials and Methods Study Site}

The Hewa Khola is one of the major tributaries of Tamor River Basin in Eastern Nepal. It is a perennial river which originates from Sattapur hill at an elevation of 3583 masl and flows from North-East to South-West direction. The Hewa Khola 'A' Hydroelectric Project (HKHEP) is a recently completed Run-of-River (RoR) project developed by the Panchthar Power Company (PPC). The project has an installed capacity of 14.9 MW and is in operation stage. The project is located in the Northeast part of Panchthar District of Eastern Development Region at a distance of about $28 \mathrm{~km}$ from the district headquarter Phidim.

The Lower Hewa Khola Hydropower Project (LHKHPP) is an under-construction RoR project that is being developed by Mountain Hydro Nepal Pvt. Ltd. The headworks of the project is located at around 600 $m$ downstream from powerhouse of HKHEP and utilizes discharge from Hewa as well as from Pheme Khola to generate 21.6 MW of rated power. The Pheme Khola is a tributary of the Tamor Basin that joins Hewa Khola at Thapatar. The location map of the projects is shown in Figure 1.

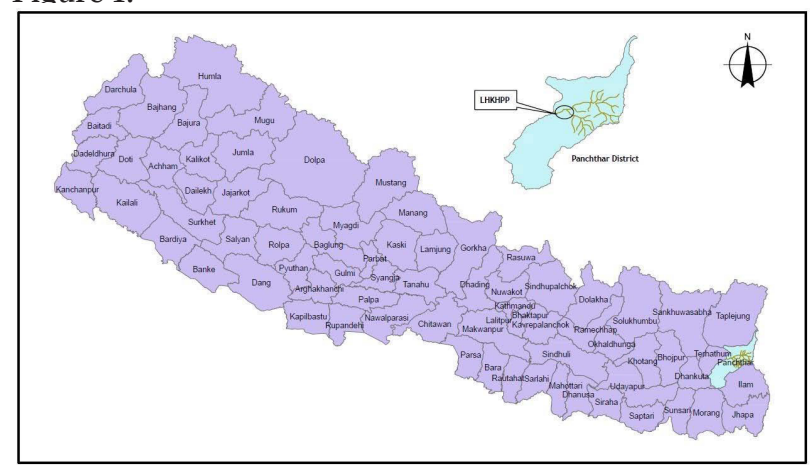

Figure 1. Location map of the projects

(Map Source: Updated Feasibility Study Report, Lower Hewa Khola HPP) 
The Eflow assessment was carried out for the dewatered zones of Hewa Khola and Pheme Khola due to construction of Hewa Khola A HEP and Lower Hewa Khola HPP. The study areas selected within the project boundaries were;

A. Site 1: In the dewatered section of Hewa Khola 'A' HEP that represents around $4.5 \mathrm{~km}$ length of river stretch from intake to powerhouse.

B. Site 2: In the dewatered stretch of Lower Hewa Khola HPP that represents around $2 \mathrm{~km}$ length between the weir of Lower Hewa Khola HPP and the confluence with Pheme Khola.

C. Site 3: In the dewatered section of Pheme Khola that represents $1.25 \mathrm{~km}$ length between Pheme Intake of Lower Hewa Khola HPP and the confluence with Hewa Khola.

D. Site 4: In the dewatered section of Hewa Khola from the confluence with Pheme Khola to the power house of Lower Hewa Khola HPP with a length of around $5 \mathrm{~km}$.

The sites selected for Eflow Assessment do not necessarily represent the actual site rather an indication of the river reach that is likely to be affected by flow diversion due to the construction of hydropower projects.

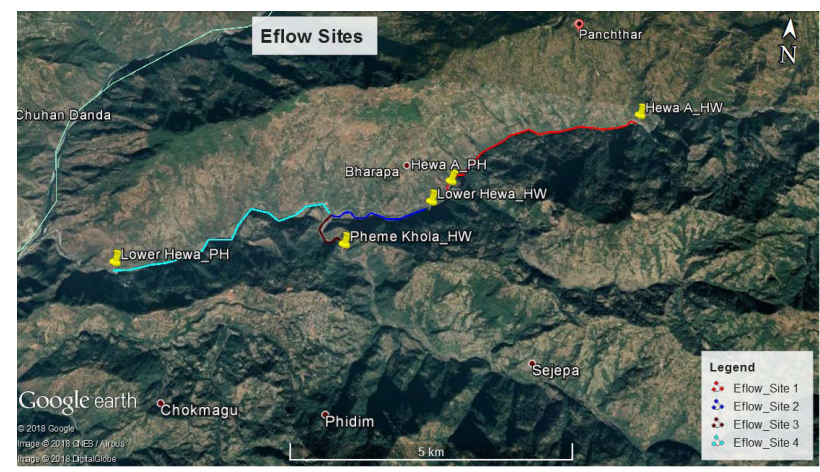

Figure 2. Study sites for the E-Flows assessment

\section{Data Collection and Processing}

Due to unavailability of sufficient data and information from the corresponding projects, hydrological data required for the study were generated using the standard method of hydrological estimation, i.e. Catchment Area Ratio method (CAR) with the recorded data from nearby gauging station no. 728. The gauging station is maintained by the Department of Hydrology and Meteorology (DHM), Government of Nepal, in Mai Khola at Rajdwali and has long term daily data record from the year 1983 to 2006.

The reliability of analysis has also been checked and compared with monthly flow adopted by the project as included in corresponding project reports. The generated flow used in this study has been converted from Gregorian to Nepali calendar in order to create a uniform base for comparison.

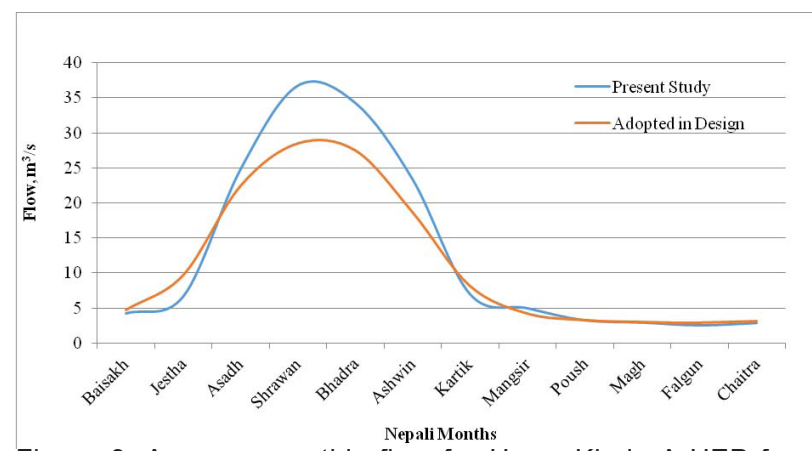

Figure 3. Average monthly flow for Hewa Khola A HEP from different studies

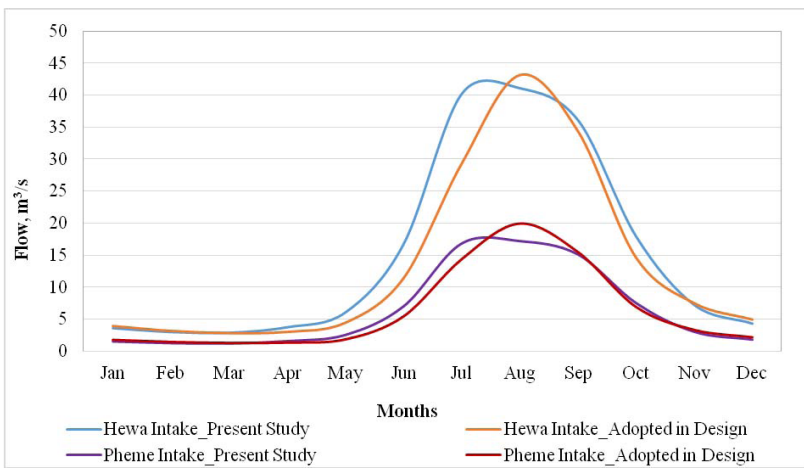

Figure 4. Average monthly flow for Lower Hewa Khola HPP from different studies

Due to unavailability of hydrological data/report regarding the projects, uniform method of analysis could not be achieved. Thus, there has been slight deviation in the long term mean monthly flow adopted by the project in design and estimated in present studies. However, the deviation being less for dry season which is of major concern for run-of-river project in general and this study in particular, the generated hydrograph from the present study has been considered reliable and thus adopted for further analysis.

Besides, two field visits were carried out during the assessment process: one for the public consultation to understand the expectations and views of relevant stakeholders on the project and the other for training and capacity building of technical staff of local bodies for discharge measurements.

\section{Data Analysis}

Modified Tennant Method is used for the Eflows Assessment and Indicators of Hydrologic Alteration (IHA) are used to define the changes in the flow indicators of the dewatered reach due to flow regulation. Instream flow regimes for ecological habitat based on Modified Tennant method used for the projects are presented in Table 1. 


\begin{tabular}{|l|c|c|}
\hline Description & $\begin{array}{l}\text { Percentage of Monthly } \\
\text { average flow }\end{array}$ & Rating or score \\
\hline $\begin{array}{l}\text { Flushing or } \\
\text { maximum }\end{array}$ & 200 & 100 \\
\hline $\begin{array}{l}\text { Optimum } \\
\text { range }\end{array}$ & $60-100$ & 100 \\
\hline Outstanding & $40-59$ & 90 \\
\hline Excellent & $30-39$ & 80 \\
\hline Good & $20-29$ & 70 \\
\hline Fair & $10-19$ & $26-50$ \\
\hline Minimum & 10 & 25 \\
\hline $\begin{array}{l}\text { Severe } \\
\text { degradation }\end{array}$ & $0-9$ & $0-20$ \\
\hline
\end{tabular}

Table 1: Instream flow regimes for ecological habitat based on Modified Tennant Method

Based on the criteria of Modified Tennant method, Eflow assessments of existing scenario for the projects were carried out.

\begin{tabular}{|c|c|c|c|c|c|c|c|}
\hline \multirow[b]{2}{*}{ Months } & \multicolumn{7}{|c|}{ Flow, $\mathrm{m}^{3} / \mathrm{s}$} \\
\hline & $\begin{array}{c}\text { River Flow } \\
\text { (mean monthly) }\end{array}$ & $\begin{array}{c}Q \text { env. (existing } \\
\text { release) }\end{array}$ & Q intake & Q spill & D/S Flow & $\begin{array}{l}\% \text { of mean } \\
\text { monthly flow }\end{array}$ & $\begin{array}{l}\text { Modified Tenant } \\
\text { Ranking Score }\end{array}$ \\
\hline Baisakh & 4.28 & 0.26 & 4.03 & 0.00 & 0.26 & $6 \%$ & Severe Degradation \\
\hline Jestha & 6.71 & 0.26 & 6.46 & 0.00 & 0.26 & $4 \%$ & Severe Degradation \\
\hline Asadh & 24.87 & 0.26 & 8.12 & 16.75 & 17.01 & $68 \%$ & Optimum Range \\
\hline Shrawan & 36.76 & 0.26 & 8.12 & 28.64 & 28.90 & $79 \%$ & Optimum Range \\
\hline Bhadra & 34.29 & 0.26 & 8.12 & 26.17 & 26.43 & $77 \%$ & Optimum Range \\
\hline Ashwin & 23.39 & 0.26 & 8.12 & 15.27 & 15.53 & $66 \%$ & Optimum Range \\
\hline Kartik & 7.03 & 0.26 & 6.77 & 0.00 & 0.26 & $4 \%$ & Severe Degradation \\
\hline Mangsir & 5.02 & 0.26 & 4.77 & 0.00 & 0.26 & $5 \%$ & Severe Degradation \\
\hline Poush & 3.30 & 0.26 & 3.04 & 0.00 & 0.26 & $8 \%$ & Severe Degradation \\
\hline Magh & 2.99 & 0.26 & 2.73 & 0.00 & 0.26 & $9 \%$ & Severe Degradation \\
\hline Falgun & 2.58 & 0.26 & 2.33 & 0.00 & 0.26 & $10 \%$ & Minimum \\
\hline Chaitra & 2.91 & 0.26 & 2.66 & 0.00 & 0.26 & $9 \%$ & Severe Degradation \\
\hline
\end{tabular}

Table 2: Assessment based on existing environmental release for Hewa Khola A HEP

\begin{tabular}{|l|c|c|c|c|c|c|c|}
\hline \multirow{2}{*}{ Months } & $\begin{array}{c}\text { River Flow } \\
\text { (mean monthly) }\end{array}$ & $\begin{array}{c}\text { Q env. (existing } \\
\text { release) }\end{array}$ & Q intake & Q spill & D/S Flow & $\begin{array}{c}\text { \% of mean } \\
\text { monthly flow }\end{array}$ & $\begin{array}{c}\text { Modified Tenant } \\
\text { Ranking Score }\end{array}$ \\
\hline Baisakh & 4.89 & 0.30 & 4.60 & 0.00 & 0.30 & $6 \%$ & Severe Degradation \\
\hline Jestha & 8.07 & 0.30 & 7.77 & 0.00 & 0.30 & $4 \%$ & Severe Degradation \\
\hline Asadh & 28.39 & 0.30 & 10.13 & 18.26 & 18.55 & $65 \%$ & Optimum Range \\
\hline Shrawan & 41.96 & 0.30 & 10.13 & 31.83 & 32.13 & $77 \%$ & Optimum Range \\
\hline Bhadra & 39.15 & 0.30 & 10.13 & 29.01 & 29.31 & $75 \%$ & Optimum Range \\
\hline Ashwin & 26.70 & 0.30 & 10.13 & 16.57 & 16.86 & $63 \%$ & Optimum Range \\
\hline Kartik & 8.46 & 0.30 & 8.16 & 0.00 & 0.30 & $3 \%$ & Severe Degradation \\
\hline Mangsir & 5.73 & 0.30 & 5.44 & 0.00 & 0.30 & $5 \%$ & Severe Degradation \\
\hline Poush & 3.77 & 0.30 & 3.47 & 0.00 & 0.30 & $8 \%$ & Severe Degradation \\
\hline Magh & 3.41 & 0.30 & 3.11 & 0.00 & 0.30 & $9 \%$ & Severe Degradation \\
\hline Falgun & 2.95 & 0.30 & 2.66 & 0.00 & 0.30 & $10 \%$ & Minimum \\
\hline Chaitra & 3.33 & 0.30 & 3.03 & 0.00 & 0.30 & $9 \%$ & Severe Degradation \\
\hline
\end{tabular}

Table 3: Assessment based on existing environmental release for Hewa Khola by Lower Hewa Khola HPP 


\begin{tabular}{|c|c|c|c|c|c|c|c|}
\hline \multirow{2}{*}{ Months } & $\begin{array}{c}\text { River Flow } \\
\text { (mean monthly) }\end{array}$ & $\begin{array}{c}\text { Q env. (existing } \\
\text { release) }\end{array}$ & Q intake & Q spill & D/S Flow & $\begin{array}{c}\text { \% of mean } \\
\text { monthly flow }\end{array}$ & $\begin{array}{c}\text { Modified Tenant } \\
\text { Ranking Score }\end{array}$ \\
\hline Baisakh & 2.04 & 0.12 & 1.92 & 0.00 & 0.12 & $6 \%$ & Severe Degradation \\
\hline Jestha & 3.59 & 0.12 & 3.47 & 0.00 & 0.12 & $3 \%$ & Severe Degradation \\
\hline Asadh & 11.87 & 0.12 & 4.71 & 7.16 & 7.28 & $61 \%$ & Optimum Range \\
\hline Shrawan & 17.54 & 0.12 & 4.71 & 12.83 & 12.96 & $74 \%$ & Optimum Range \\
\hline Bhadra & 16.36 & 0.12 & 4.71 & 11.66 & 11.78 & $72 \%$ & Optimum Range \\
\hline Ashwin & 11.16 & 0.12 & 4.71 & 6.45 & 6.58 & $59 \%$ & Optimum Range \\
\hline Kartik & 3.77 & 0.12 & 3.65 & 0.00 & 0.12 & $3 \%$ & Severe Degradation \\
\hline Mangsir & 2.40 & 0.12 & 2.27 & 0.00 & 0.12 & $5 \%$ & Severe Degradation \\
\hline Poush & 1.57 & 0.12 & 1.45 & 0.00 & 0.12 & $8 \%$ & Severe Degradation \\
\hline Magh & 1.43 & 0.12 & 1.30 & 0.00 & 0.12 & $9 \%$ & Severe Degradation \\
\hline Falgun & 1.23 & 0.12 & 1.11 & 0.00 & 0.12 & $10 \%$ & Minimum \\
\hline Chaitra & 1.39 & 0.12 & 1.27 & 0.00 & 0.12 & $9 \%$ & Severe Degradation \\
\hline
\end{tabular}

Table 4: Assessment based on existing environmental release for Pheme Khola by Lower Hewa Khola HPP.

The Eflow assessment for the projects is based on different scenarios as described in Table 5 .

\begin{tabular}{|c|c|c|c|}
\hline Scenarios & Code & Description & Remarks \\
\hline $\begin{array}{l}\text { Baseline } \\
\text { Scenario }\end{array}$ & 10\%_MinM & $\begin{array}{l}10 \% \text { of } \\
\mathrm{m} \text { i } \mathrm{n} \text { i } \mathrm{m} \text { m } \\
\mathrm{m} \text { o } \mathrm{n} \mathrm{h} \text { l y } \\
\text { average flow }\end{array}$ & $\begin{array}{l}\text { E x i s t i n g } \\
\text { environmental } \\
\text { release for the } \\
\text { project }\end{array}$ \\
\hline Scenario I & 10\%_MeanM & $\begin{array}{l}10 \% \text { of mean } \\
\text { monthly flow }\end{array}$ & $\begin{array}{l}\text { Considered } \\
\text { in this } \\
\text { assessment }\end{array}$ \\
\hline Scenario II & 15\%_MinM & $\begin{array}{l}15 \% \text { of } \\
\mathrm{m} \text { i } \mathrm{n} \text { i } \mathrm{m} \text { m } \\
\mathrm{m} \text { o } \mathrm{n} \mathrm{h} \text { I y } \\
\text { average flow }\end{array}$ & $\begin{array}{l}\text { Considered } \\
\text { in this } \\
\text { assessment }\end{array}$ \\
\hline $\begin{array}{l}\text { Scenario } \\
\text { III }\end{array}$ & 20\%_MinM & $\begin{array}{l}20 \% \text { of } \\
\mathrm{m} \text { i } \mathrm{n} \text { i } \mathrm{m} \text { m } \\
\mathrm{m} \text { o } \mathrm{n} \mathrm{h} \text { I y } \\
\text { average flow }\end{array}$ & $\begin{array}{l}\text { Considered } \\
\text { in this } \\
\text { assessment }\end{array}$ \\
\hline $\begin{array}{l}\text { Scenario } \\
\text { IV }\end{array}$ & 30\%_MinM & $\begin{array}{l}30 \% \text { of } \\
\mathrm{m} \text { i } \mathrm{n} \mathrm{i} \mathrm{m} \mathrm{u} \mathrm{m} \\
\mathrm{m} \text { o } \mathrm{n} \mathrm{th} \mathrm{I} \mathrm{y} \\
\text { average flow }\end{array}$ & $\begin{array}{l}\text { Considered } \\
\text { in this } \\
\text { assessment }\end{array}$ \\
\hline
\end{tabular}

Table 5: Scenarios selected for assessment

\section{Results}

Based on scenarios defined in Table 5, an assessment of E-flow for the projects at study sites described above were carried out and presented in Figure 5 to Figure 8.

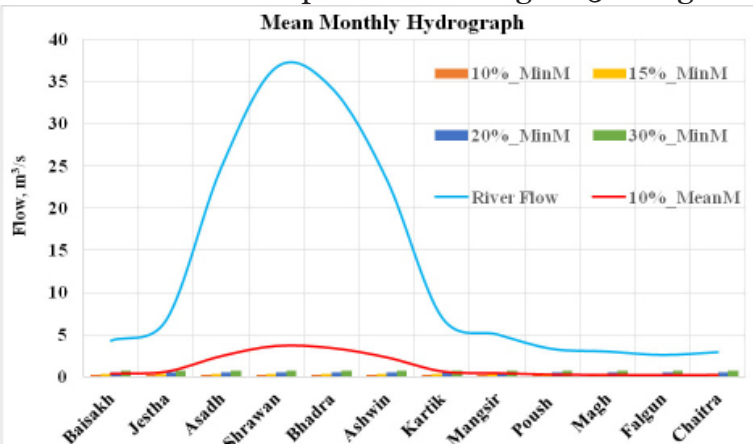

Figure 5. Mean monthly hydrograph for Site 1 at different flow release scenarios

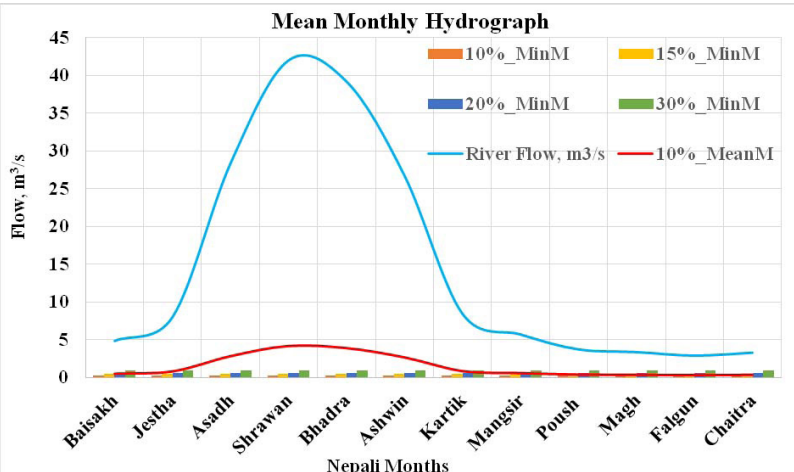

Figure 6. Mean monthly hydrograph for Site 2 at different flow release scenarios

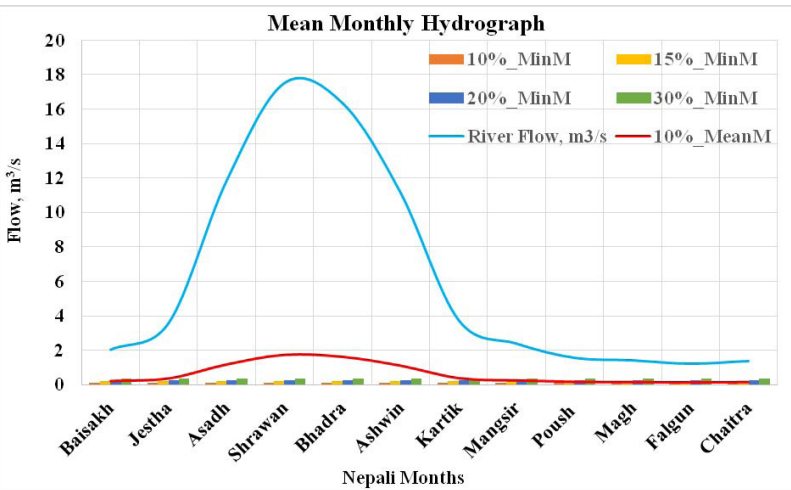

Figure 7. Mean monthly hydrograph for Site 3 at different flow release scenarios

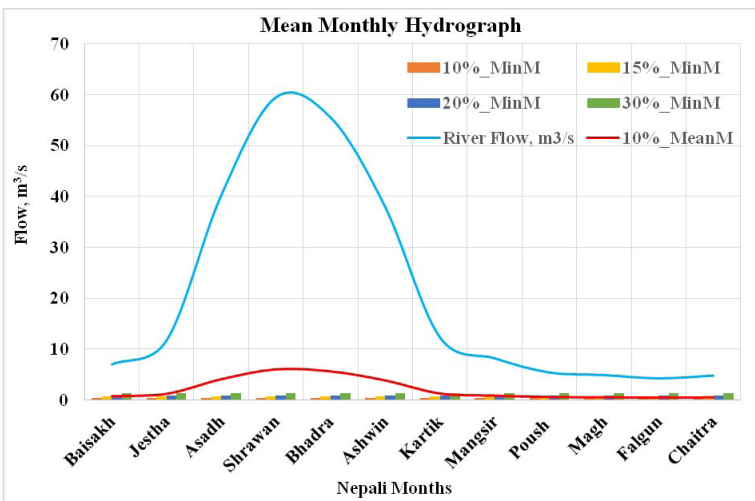

Figure 8. Mean monthly hydrograph for Site 4 at different flow release scenarios 


\begin{tabular}{|c|c|c|c|c|c|c|c|}
\hline \multicolumn{1}{|c|}{ Months } & \multicolumn{1}{|c|}{$\begin{array}{c}\text { River } \\
\text { Flow }\end{array}$} & $\begin{array}{c}\text { Q env. } \\
\text { (existing } \\
\text { release) }\end{array}$ & $\begin{array}{c}\text { D/S } \\
\text { Flow }\end{array}$ & $\begin{array}{c}\text { \% of } \\
\text { natural } \\
\text { flow }\end{array}$ & $\begin{array}{c}\mathbf{2 0} \% \text { of } \\
\text { monthly } \\
\text { average flow }\end{array}$ & $\begin{array}{c}\text { Additional flow required } \\
\text { in D/S for achieving good } \\
\text { condition }\end{array}$ & $\begin{array}{c}\text { Total Downstream } \\
\text { Flow Required } \\
\text { (Proposed Eflow) }\end{array}$ \\
\hline Baisakh & 4.28 & 0.26 & 0.26 & $6 \%$ & 0.86 & 0.60 & 0.86 \\
\hline Jestha & 6.71 & 0.26 & 0.26 & $4 \%$ & 1.34 & & 1.34 \\
\hline Asadh & 24.87 & 0.26 & 17.01 & $68 \%$ & 4.97 & & 17.01 \\
\hline Shrawan & 36.76 & 0.26 & 28.90 & $79 \%$ & 7.35 & & 28.90 \\
\hline Bhadra & 34.29 & 0.26 & 26.43 & $77 \%$ & 6.86 & & 26.43 \\
\hline Ashwin & 23.39 & 0.26 & 15.53 & $66 \%$ & 4.68 & & 15.53 \\
\hline Kartik & 7.03 & 0.26 & 0.26 & $4 \%$ & 1.41 & & 1.41 \\
\hline Mangsir & 5.02 & 0.26 & 0.26 & $5 \%$ & 1.00 & & 1.00 \\
\hline Poush & 3.30 & 0.26 & 0.26 & $8 \%$ & 0.66 & 0.15 & 0.66 \\
\hline Magh & 2.99 & 0.26 & 0.26 & $9 \%$ & 0.60 & 0.40 & 0.60 \\
\hline Falgun & 2.58 & 0.26 & 0.26 & $10 \%$ & 0.52 & 0.34 & 0.52 \\
\hline Chaitra & 2.91 & 0.26 & 0.26 & $9 \%$ & 0.58 & 0.26 & 0.58 \\
\hline
\end{tabular}

Table 6: Environmental Flow Regime for Hewa Khola A HEP

\begin{tabular}{|c|c|c|c|c|c|c|c|c|c|}
\hline \multirow[b]{2}{*}{ Months } & \multicolumn{9}{|c|}{ Flow, $\mathrm{m}^{3} / \mathrm{s}$} \\
\hline & $\begin{array}{l}\text { River } \\
\text { Flow }\end{array}$ & $\begin{array}{l}\text { Q env. } \\
\text { (exist- } \\
\text { ing re- } \\
\text { lease) }\end{array}$ & $\begin{array}{l}\text { D/S } \\
\text { Flow }\end{array}$ & $\begin{array}{c}\% \text { of } \\
\text { natural } \\
\text { flow }\end{array}$ & $\begin{array}{l}20 \% \text { of } \\
\text { monthly } \\
\text { average } \\
\text { flow }\end{array}$ & $\begin{array}{c}\text { Additional } \\
\text { flow required } \\
\text { in } \mathrm{D} / \mathrm{S} \text { for } \\
\text { achieving good } \\
\text { condition }\end{array}$ & $\begin{array}{l}\text { Total } \\
\text { Down- } \\
\text { stream } \\
\text { Flow } \\
\text { Required }\end{array}$ & $\begin{array}{l}\text { Flow required } \\
\text { for Irrigation in } \\
\text { the Dewatered } \\
\text { Section (men- } \\
\text { tioned in the PPA } \\
\text { Agreement) }\end{array}$ & $\begin{array}{l}\text { Proposed } \\
\text { E-flow } \\
\text { for Lower } \\
\text { Hewa } \\
\text { HPP }\end{array}$ \\
\hline Baisakh & 6.94 & 0.42 & 0.42 & $6 \%$ & 1.39 & 0.97 & 1.39 & 0.02 & 1.41 \\
\hline Jestha & 11.66 & 0.42 & 0.42 & $4 \%$ & 2.33 & 1.91 & 2.33 & 0.02 & 2.35 \\
\hline Asadh & 40.25 & 0.42 & 25.83 & $64 \%$ & 8.05 & & 25.83 & 0.02 & 25.85 \\
\hline Shrawan & 59.50 & 0.42 & 45.08 & $76 \%$ & 11.90 & & 45.08 & 0.02 & 45.10 \\
\hline Bhadra & 55.51 & 0.42 & 41.09 & $74 \%$ & 11.10 & & 41.09 & 0.02 & 41.11 \\
\hline Ashwin & 37.86 & 0.42 & 23.44 & $62 \%$ & 7.57 & & 23.44 & 0.02 & 23.46 \\
\hline Kartik & 12.23 & 0.42 & 0.42 & $3 \%$ & 2.45 & 2.03 & 2.45 & 0.02 & 2.47 \\
\hline Mangsir & 8.13 & 0.42 & 0.42 & $5 \%$ & 1.63 & 1.21 & 1.63 & 0.02 & 1.65 \\
\hline Poush & 5.34 & 0.42 & 0.42 & $8 \%$ & 1.07 & 0.65 & 1.07 & 0.02 & 1.09 \\
\hline Magh & 4.83 & 0.42 & 0.42 & $9 \%$ & 0.97 & 0.55 & 0.97 & 0.02 & 0.99 \\
\hline Falgun & 4.18 & 0.42 & 0.42 & $10 \%$ & 0.84 & 0.42 & 0.84 & 0.02 & 0.86 \\
\hline Chaitra & 4.72 & 0.42 & 0.42 & $9 \%$ & 0.94 & 0.53 & 0.94 & 0.02 & 0.96 \\
\hline
\end{tabular}

Table 7: Environmental Flow Regime for Lower Hewa Khola HPP

Based on the analysis of the different scenarios, the Eflow assessments for the projects are defined so as to achieve at least good ecological condition in the dewatered reach according to Modified Tennant Method. That is to say, the flow in the dewatered reach has to be at least $20 \%$ of the mean monthly average flow.

The requirements of the downstream water use such as irrigation requirements, cremation sites and riparian releases have been considered in the assessment. There is not much irrigated land in the dewatered zone of Hewa Khola A HEP and the discharge from the tributaries and occasional release from the intake are expected to fulfill the requirements for irrigation and other uses. Considering these factors in assessment, the Eflow defined for Hewa Khola A HEP is presented in Table 6.
However, in case of Lower Hewa Khola HPP, there is already a provision for releasing $0.02 \mathrm{~m}^{3} / \mathrm{s}$ of water every month in the Power Purchase Agreement (PPA) for meeting irrigation water requirements in the dewatered reach. However, during the consultation meeting in the field, local people mentioned that about 5000 Ropani (Approximately 255 hectares) of land is irrigated and 3 crops per year are grown in the fields. This might necessitate release of more than $0.02 \mathrm{~m}^{3} / \mathrm{s}$ of water which has not been included in the recommended Eflow value for Lower Hewa Khola HPP. Furthermore, minimum flow release, to be made by the developer, is expected to meet the requirements for cultural purpose ${ }^{1}$.

Thus, based on available information and assessment, the Eflow defined for Lower Hewa HPP is shown in Table 7. As the dewatered reach of the Pheme Khola up to the 
confluence with Hewa Khola is about $1 \mathrm{~km}$, combined Eflow for Lower Hewa Khola HPP (Hewa Khola+Pheme Khola) has been defined, which is a reasonable approximation based on the project's capacity and the overall dewatered stretch considered.

\section{Results and Discussions \\ Effects on power production}

Based on scenarios defined in Table 5, the effect of different levels of E-Flow releases on energy production was evaluated for the projects. Following assumptions were made for energy calculations:

- Fixed amount of head loss for different flow conditions, i.e., $4 \%$ of the gross head.

- Overall efficiency of $87.4 \%$ for Hewa Khola A and 89.3\% for Lower Hewa Khola HPP.

- Energy Outages of $4 \%$.

- Revenue generation based on;

- Dry season energy: Poush to Chaitra @ 8.40 $\mathrm{NRs} / \mathrm{kWh}$

- Wet Season Energy: Remaining months@ @4.80 $\mathrm{NRs} / \mathrm{kWh}$

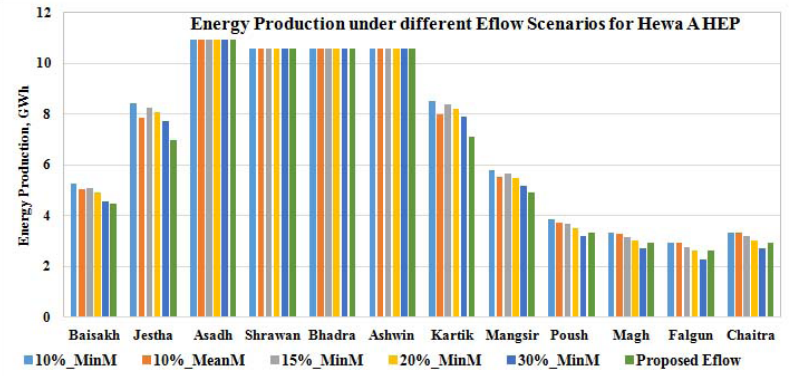

Figure 9. Energy production for Hewa Khola A HEP under different Eflow scenarios

Similarly, energy calculation for Lower Hewa Khola HPP was also carried out for different Eflow scenarios in Hewa Khola as well as Pheme Khola. The combined discharge available after environmental release as well as release for irrigation purpose has been used in calculation of power and energy for the project.

\begin{tabular}{|c|c|c|c|c|c|c|}
\hline \multicolumn{7}{|c|}{ Energy Production (GWh) } \\
\hline Eflow Scenarios & Base Scenario & Scenario I & Scenario II & Scenario III & Scenario IV & $\begin{array}{l}\text { E-flow } \\
\text { Scenario }\end{array}$ \\
\hline Months & 10\%_Min M & 10\%_MeanM & 15\%_MinM & 20\%_MinM & 30\%_MinM & Proposed \\
\hline Baisakh & 5.24 & 5.02 & 5.08 & 4.91 & 4.57 & 4.46 \\
\hline Jestha & 8.41 & 7.87 & 8.24 & 8.07 & 7.74 & 7.00 \\
\hline Asadh & 10.92 & 10.92 & 10.92 & 10.92 & 10.92 & 10.92 \\
\hline Shrawan & 10.58 & 10.58 & 10.58 & 10.58 & 10.58 & 10.58 \\
\hline Bhadra & 10.58 & 10.58 & 10.58 & 10.58 & 10.58 & 10.58 \\
\hline Ashwin & 10.58 & 10.58 & 10.58 & 10.58 & 10.58 & 10.58 \\
\hline Kartik & 8.54 & 7.98 & 8.37 & 8.21 & 7.88 & 7.09 \\
\hline Mangsir & 5.81 & 5.51 & 5.65 & 5.49 & 5.18 & 4.90 \\
\hline Poush & 3.83 & 3.74 & 3.67 & 3.51 & 3.18 & 3.33 \\
\hline Magh & 3.32 & 3.28 & 3.17 & 3.01 & 2.69 & 2.91 \\
\hline Falgun & 2.93 & 2.93 & 2.77 & 2.61 & 2.28 & 2.61 \\
\hline Chaitra & 3.35 & 3.31 & 3.19 & 3.02 & 2.70 & 2.94 \\
\hline Total & 84.1 & 82.3 & 82.8 & 81.5 & 78.9 & 77.9 \\
\hline Dry Season & 13.4 & 13.3 & 12.8 & 12.1 & 10.9 & 11.79 \\
\hline Wet Season & 70.6 & 69.0 & 70.0 & 69.3 & 68.0 & 66.09 \\
\hline $\begin{array}{l}\begin{array}{l}\mathbf{R} \text { e v e } \\
\text { Generation, }\end{array} \\
\text { NPR }\end{array}$ & 657.9 & 643.4 & 649.3 & 640.6 & 623.4 & 611.75 \\
\hline $\begin{array}{l}\text { Dry } \\
\text { Generation, mill. NPR }\end{array}$ & 64.5 & 63.6 & 61.4 & 58.3 & 52.1 & 56.57 \\
\hline $\begin{array}{lr}\text { Wet } & \text { Season } \\
\text { Generation, mill. NPR }\end{array}$ & 593.4 & 579.8 & 587.9 & 582.3 & 571.3 & 555.18 \\
\hline $\begin{array}{l}\text { \% Change in } \\
\text { Energy }\end{array}$ & $0 \%$ & $-2 \%$ & $-2 \%$ & $-3 \%$ & $-6 \%$ & $-7 \%$ \\
\hline $\begin{array}{l}\text { Total Loss of } \\
\text { Revenue, mill. NPR }\end{array}$ & $\mathbf{0}$ & -14 & -9 & -17 & -34 & -46 \\
\hline
\end{tabular}

Table 8: Energy production for Hewa Khola A HEP under different E-flow scenarios 


\begin{tabular}{|c|c|c|c|c|c|c|}
\hline \multicolumn{7}{|c|}{ Energy Production (GWh) } \\
\hline E-flow Scenarios & $\begin{array}{c}\text { Base } \\
\text { Scenario }\end{array}$ & Scenario I & Scenario II & Scenario III & Scenario IV & $\begin{array}{c}\text { E-flow } \\
\text { Scenario }\end{array}$ \\
\hline Months & 10\%_MinM & 10\%_MeanM & 15\%_MinM & 20\%_MinM & 30\%_MinM & Proposed \\
\hline Baisakh & 6.76 & 6.48 & 6.55 & 6.33 & 5.89 & 5.75 \\
\hline Jestha & 11.68 & 10.90 & 11.46 & 11.25 & 10.81 & 9.69 \\
\hline Asadh & 15.93 & 15.93 & 15.93 & 15.93 & 15.93 & 15.95 \\
\hline Shrawan & 15.43 & 15.43 & 15.43 & 15.43 & 15.43 & 15.45 \\
\hline Bhadra & 15.43 & 15.43 & 15.43 & 15.43 & 15.43 & 15.45 \\
\hline Ashwin & 15.43 & 15.43 & 15.43 & 15.43 & 15.43 & 15.45 \\
\hline Kartik & 11.88 & 11.07 & 11.67 & 11.46 & 11.03 & 9.83 \\
\hline Mangsir & 7.49 & 7.11 & 7.29 & 7.09 & 6.68 & 6.32 \\
\hline Poush & 4.94 & 4.82 & 4.73 & 4.52 & 4.10 & 4.28 \\
\hline Magh & 4.28 & 4.22 & 4.08 & 3.87 & 3.47 & 3.75 \\
\hline Falgun & 3.77 & 3.77 & 3.56 & 3.35 & 2.93 & 3.35 \\
\hline Chaitra & 4.31 & 4.26 & 4.10 & 3.89 & 3.47 & 3.78 \\
\hline Total & 117.3 & 114.8 & 115.6 & 114.0 & 110.6 & 109.0 \\
\hline Dry Season & 17.3 & 17.1 & 16.5 & 15.6 & 14.0 & 15.16 \\
\hline Wet Season & 100.0 & 97.8 & 99.2 & 98.3 & 96.6 & 93.89 \\
\hline $\begin{array}{l}\text { Revenue Generation, } \\
\text { mill. NPR }\end{array}$ & 923.3 & 903.2 & 912.1 & 901.0 & 878.7 & 861.43 \\
\hline $\begin{array}{l}\text { Dry Season Generation, } \\
\text { mill. NPR }\end{array}$ & 83.1 & 82.0 & 79.1 & 75.1 & 67.0 & 72.79 \\
\hline $\begin{array}{l}\text { Wet Season Generation, } \\
\text { mill. NPR }\end{array}$ & 840.2 & 821.2 & 833.1 & 825.9 & 811.6 & 788.6 \\
\hline \% Change in Energy & $0 \%$ & $-2 \%$ & $-1 \%$ & $-3 \%$ & $-6 \%$ & $-7 \%$ \\
\hline $\begin{array}{l}\text { Total Loss of Revenue, } \\
\text { mill. NPR }\end{array}$ & 0 & -20 & -11 & -22 & -45 & -62 \\
\hline
\end{tabular}

Table 9: Energy production for Lower Hewa Khola HPP under different Eflow scenarios

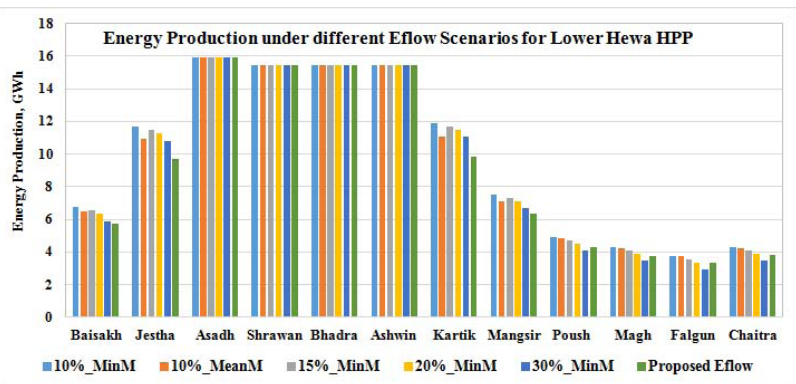

Figure 10. Energy production for Lower Hewa Khola HPP under different Eflow scenarios

The effect of proposed Eflow for Hewa Khola A HEP and Lower Hewa Khola HPP in terms of loss of annual energy and annual revenue has been depicted in Table 8 and Table 9, and in Figure 9 and Figure 10. The effect is significant during the dry season when there is low flow in the rivers and the rate per unit of energy is high.

Energy production decreases in order of increasing EFlow releases. Similarly, the revenue loss also increases with increase in Eflow release. Hewa Khola A HEP being a recently completed project, loss in revenue from additional release of environmental flow may not be economical to adopt. However, the loss in revenue against environmental provision is only $7 \%$ for the proposed Eflow Scenario compared to the base scenario for both the projects.

\section{Social and Environmental Conflicts}

Based on field observation of the project area and discussion with the local stakeholders, pessimistic situation was revealed at site regarding downstream releases. Despite the regulation to release at least 10\% of minimum monthly average flow throughout the year as the minimum release, both the projects were found with no structural provision in intake for such releases. This shows that the developers are reluctant to comply with the minimum flow release criterion set by the regulatory body. As a result, social conflicts may arise in future.

Fish passage usually acts as a suitable means for migration of fish against the barrier effect created by the weir/dam in many hydropower projects. Eflows can be released through fish passage structures based on their design. However, there is no provision of fish passage in both of these hydropower projects due to which connectivity will be lost completely for upstream migration of fish, if not for downstream.

\section{Conclusions}

The Eflow Assessment of Hewa Khola A HEP and Lower Hewa Khola HPP conducted under this study indicate that the projects are likely to create significant impacts in the dewatered reach and affect the riverine ecosystem. Six Eflows scenarios were evaluated including the proposed Eflow at four Eflows sites.

The results of the Eflows assessment unsurprisingly conclude that the best Eflows scenario for the projects is the release of more water during the winter (low flow) 
months. However, power generation will be negatively impacted with the release of more water. Given that the Hewa Khola A HEP is already in operation and the Lower Hewa Khola HPP is under construction, inclusion of minimum flow release mechanism in the intake structure is required. Similarly, provision of sufficient water for irrigation in the dewatered zone of Lower Hewa Khola HPP is essential. This is to ensure cooperation between the developer and the local stakeholders and reduce social and environmental conflicts that may otherwise arise.

Thus, the environmental flow shall be released in the dewatered stretch of the river, thereby, ensuring cooperation among the concerned stakeholders and sustainable hydropower development in the basin. In order to avoid future conflicts and to bring uniformity in the planning and implementation phase, a basin wide Eflows Assessment with greater stakeholder participation is recommended.

\section{Acknowledgement}

Research for this article was funded by the Netherlands Organization for Scientific Research and the Department for International Development. It was conducted as a part of the project 'Hydropower Development in the context of Climate Change - Exploring Conflicts and Fostering Cooperation across Scales and Boundaries in the Eastern Himalaya' (file no. W. 07.68.413).

Narayanhari Rijal holds master's degree in Hydropower Development from Norwegian University of Science and Technology (NTNU), Norway. He is currently working in Paragon Engineering Consultancy and Research Centre Pvt. Ltd., Nepal as the Chairperson and is involved in management of various infrastructure projects undertaken by the firm. His areas of research interests are Environmental Impacts of hydropower projects and Ecological modeling.

Corresponding E-mail:narayanin2004@gmail.com
Hari Krishna Shrestha, PhD, is a Professor at Nepal Engineering College. He is currently working as the Principal of the College.

E-mail: hari@nec.edu.np.

Bert Bruins is lecturer at the Water Resources Management Group of Wageningen University and Research, the Netherlands. He is the coordinator the CCMCC-Hydropower project.

E-mail:bert.bruins@wur.nl

\section{Bibliography}

Angel Engineering Consultancy, Hydrological Report: Lower Hewa Khola HPP, Page 2

Mountain Energy Limited, Updated Feasibility Study Report: Lower Hewa Khola HPP

Panchthar Power Company (2010). Final IEE Report, Hewa Khola A Hydroelectric Project

RICHTER, B. D., BAUMGARTNER, J. V., POWELL, J. \& BRAUN, D. P. 1996. A method for assessing hydrologic alteration within ecosystems. Conservation biology, 10, 1163-1174.

Sanima Hydro and Engineering Pvt.Ltd.(2018) retrieved on 31st March 2018: http://sanimaengineering. $\mathrm{com} /$ project/hewa-khola-a-hydroelectric-project/

\section{Footnotes}

1. Due to the lack of complete information regarding the abundance and diversity of fish species, habitat information and flow hydraulics, detail calculations of flow requirements for fish species have not been made. This requires a better assessment demanding more time and budget. 